\title{
Surgeon Perspectives about Local Therapy for Breast Carcinoma
}

\author{
Steven J. Katz, M.D., M.P... ${ }^{1-3}$ \\ Paula M. Lantz, Ph.D. ${ }^{2}$ \\ Nancy K. Janz, Ph.D. ${ }^{4}$ \\ Angela Fagerlin, Ph.D. ${ }^{1,3}$ \\ Kendra Schwartz, M.D., M.S.P.H. ${ }^{5,6}$ \\ Lihua Liu, Ph.D. ${ }^{7}$ \\ Dennis Deapen, Dr.P.H. ${ }^{7}$ \\ Barbara Salem, M.S.w. ${ }^{1}$ \\ Indu Lakhani, M.s. ${ }^{1}$ \\ Monica Morrow, M.D. ${ }^{8}$

\begin{abstract}
${ }^{1}$ Division of General Medicine, Department of Internal Medicine, University of Michigan, Ann Arbor, Michigan. 2 Department of Health Management and Policy,
University of Michigan, Ann Arbor, Michigan.
\end{abstract}

${ }^{3}$ Veterans Affairs Ann Arbor Healthcare System, Ann Arbor, Michigan.

${ }^{4}$ Department of Health Behavior and Health Education, University of Michigan, Ann Arbor, Michigan.

${ }^{5}$ Department of Family Medicine, Wayne State University, Detroit, Michigan.

${ }^{6}$ Karmanos Cancer Institute, Wayne State University, Detroit, Michigan.

${ }^{7}$ Department of Preventive Medicine, Keck School of Medicine, University of Southern California, Los Angeles, California.

${ }^{8}$ Department of Surgical Oncology, Fox Chase Cancer Center, Philadelphia, Pennsylvania.

Supported by Grant R01 CA8837-A1 from the National Cancer Institute to the University of Michigan, and by Federal funds from the National Cancer Institute, National Institutes of Health, Department of Health and Human Services, under Contract No. N01-PC-35139 and N01-PC-65064.

The authors thank the American College of Surgeons Breast Cancer Department (Connie Bura and David Winchester M.D.) for their support.

The collection of cancer incidence data used in the current study was supported by the California Department of Health Services as part of the state-

\begin{abstract}
BACKGROUND. Geographic variations in the use of mastectomy and the use of radiation therapy (RT) after breast-conserving surgery (BCS) have motivated concerns that surgeons are not uniformly adhering to treatment standards.

METHODS. The authors surveyed attending surgeons of a population-based sample of patients with breast carcinoma diagnosed in Detroit and Los Angeles from December 2001 to January 2003 ( $n=365$; response rate, 80.0\%). Clinical scenarios were used to evaluate opinions about local therapy.
\end{abstract}

RESULTS. On average, surgeons reported that they devoted $31.3 \%$ of their total practice to breast carcinoma. Approximately one-half of surgeons practiced in a community hospital setting, whereas $18.8 \%$ practiced in a cancer center. Compared to low volume surgeons, high volume surgeons were more likely to favor BCS with RT for invasive breast carcinoma $(60.8 \%, 74.0 \%$, and $87.2 \%$ for low, moderate, and high volume surgeons, respectively, $P<0.001$ ). Surgeons who favored BCS were more likely to perceive greater quality of life (QOL) benefits for BCS than mastectomy (85.9\%) compared with surgeons who favored mastectomy (28.6\%) and those who did not favor 1 procedure over the other $(60.0 \%, P<0.001)$. In a ductal carcinoma in situ scenario, $35.0 \%$ of surgeons favored BCS without RT and $61.0 \%$ favored BCS with RT. Opinions regarding the role of RT after BCS varied by geographic site, surgeon volume, and patient age.

CONCLUSIONS. Variation in surgeon opinion concerning local therapy reflected clinical uncertainty about the benefits of alternative treatments. High volume surgeons more frequently endorsed current clinical guidelines that favor BCS compared with mastectomy. This may partly be explained by the greater belief that BCS confers a better patient QOL than mastectomy. Cancer 2005;104:1854-61. () 2005 American Cancer Society.

KEYWORDS: surgeon perspectives, local therapy, breast carcinoma, breast-conserving surgery.

arge geographic variations in both the use of mastectomy for - treatment of early-stage breast carcinoma ${ }^{1-8}$ and the use of radiation therapy (RT) after breast-conserving surgery $(\mathrm{BCS})^{1,4,8-10}$ have

wide cancer reporting program mandated by California Health and Safety Code Section 103885.

Address for reprints: Steven J. Katz, M.D., M.P.H., Department of Health Management and Policy, University of Michigan, $300 \mathrm{~N}$. Ingalls, Suite 7E12, P. 0. Box 0429, Ann Arbor, Ml 48109-0429; Fax: (734) 936-8944; E-mail: skatz@umich.edu
The ideas and opinions expressed herein are those of the author, and no endorsement by the State of California, Department of Health Services, is intended or should be inferred.

Received March 29, 2005; revision received April 29, 2005; accepted May 31, 2005. 
motivated concerns about the quality of breast carcinoma treatment in the U.S. Mastectomy is believed to represent overtreatment in many cases, whereas the omission of RT after BCS is considered to be evidence of undertreatment. These observations have motivated policy makers and some clinicians to suggest that surgeons are not uniformly adhering to treatment standards. ${ }^{2,6,8,9,11}$

Although surgeons have enormous influence on decisions about local therapy for breast carcino$\mathrm{ma}{ }^{10,12-16}$, our understanding of surgeons' knowledge and attitudes toward treatment options is limited. Previous studies have been limited by small samples, low response rates, a focus on a single treatment issue (e.g., reconstruction after mastectomy), or the use of non-U.S. samples. ${ }^{5,17-19}$ One large study performed by Mandelblatt et al. in $1998^{20}$ evaluated correlates of surgeons' opinions about surgical treatment for elderly patients with invasive breast carcinoma. Surgeon attitudes favoring BCS were associated with a higher breast surgery volume, being female, and region of the country.

Breast surgery is performed by many general surgeons and wide variations in surgical experience and practice settings may have important clinical implications. To address whether these variations are associated with surgeons' perspectives about treatment issues, we surveyed surgeons who performed breast surgery in the metropolitan areas of Detroit and Los Angeles in 2002. The aims of the study were to evaluate surgeons' perspectives regarding surgical and adjuvant RT options for women with breast carcinoma and to determine whether surgeon characteristics or practice setting were associated with knowledge and attitudes toward surgical treatment options for patients with breast carcinoma.

\section{MATERIALS AND METHODS \\ Study Sample}

We performed a survey of a population-based sample of 2645 women with breast carcinoma diagnosed in the metropolitan areas of Detroit and Los Angeles during the study period from December 2001 to January 2003. The details of the current study are reported elsewhere. ${ }^{21}$ In brief, all patients with ductal carcinoma in situ (DCIS) and an approximate 20\% random sample of patients with invasive (but not metastatic) disease were accrued into the sample during the study period. Pathology reports were used to identify $\geq 1$ surgeon $(n=456)$ for $98.5 \%$ of the patient sample.

Surgeons were contacted by mail and asked to participate in a brief, self-administered survey to evaluate their perspectives about surgical treatment for breast carcinoma. Surgeons were subsequently mailed a questionnaire and gift. We followed the Dillman method-which involved a postcard reminder and subsequent mailings to nonresponders-to maximize response rates in a mailed survey. ${ }^{22,23}$ A telephone interview was requested for surgeons who did not respond to the mailed survey. The response rate was 80.0\% ( $n=365)$ : 355 surgeons completed the written questionnaire and 10 surgeons completed an abbreviated telephone survey. Surgeons were informed of study goals and methods and the protocol was approved by the institutional review boards of the University of Michigan, Wayne State University, and the University of Southern California.

\section{Measures}

The questionnaire was developed based on a conceptual model and extensive pilot testing. The content included questions pertaining to demographics (age, gender, yrs in practice since residency), practice patterns (the percentage of total practice time devoted to breast carcinoma-related procedures, other oncologyrelated and nononcology-related procedures, and the number of definitive breast carcinoma surgery procedures per year), and hospital practice setting (cancer center, teaching hospital, community hospital). A variable, relative breast surgery practice volume, was created by recoding the percentage of total practice time devoted to breast carcinoma-related surgery into 3 categories (approximately tertiles): low volume ( $\leq$ $15 \%$ of total practice), moderate volume (16-38\%), and high volume $(>38 \%)$. Varying the cutoff ranges did not change the conclusions in the current study. We chose this measure rather than surgeon recall of the number of procedures performed per year because there were 13 fewer missing observations. However, the same results were observed when data were analyzed with number of procedures as the independent variable.

We included two clinical scenarios to evaluate surgeons' knowledge, attitudes, and preferences toward breast surgery treatment options.

Scenario 1 (invasive disease) described a (40- or 65)year-old woman (who) detects a mass and is referred to you for evaluation. A physical examination demonstrates a suspicious mass $2 \mathrm{~cm}$ in diameter. There are no palpable lymph nodes. Subsequent biopsy reveals invasive ductal carcinoma.

Scenario 2 (DCIS) described a (40- or 65-)year-old woman (who) has a suspicious screening mammogram which yields a subsequent diagnosis of a small unicentric low-grade DCIS.

Patient age, either 40 or 65 years old, was randomly assigned in a $2 \times 2$ factorial design. Surgeons 
TABLE 1

Study Sample Characteristics

\begin{tabular}{lll}
\hline Variables & $\begin{array}{l}\text { No. of } \\
\text { surgeons }\end{array}$ & Percent \\
\hline Mean age in yrs, (range) & 365 & $49.4(30-79)$ \\
Female & 53 & 14.4 \\
Male & 312 & 85.6 \\
Mean yrs in practice, (range) & 365 & $17.2(1-49)$ \\
Mean percent breast carcinoma (range) & 365 & $31.3(2-100)$ \\
Percent other oncology (range) & 365 & $16.2(0-70)$ \\
Percent non-oncology (range) & 365 & $52.5(0-95)$ \\
No. of breast surgeries per yr & & \\
$\quad \leq 10$ & 42 & 11.5 \\
11-20 & 62 & 17.0 \\
21-50 & 135 & 37.0 \\
51-100 & 62 & 17.0 \\
$>100$ & 51 & 14.0 \\
Missing & 13 & 3.5 \\
Hospital practice setting & & \\
Community hospital & 176 & 48.2 \\
Teaching hospital & 120 & 33.0 \\
Cancer center & 69 & 18.8 \\
\hline
\end{tabular}

were asked three questions after each scenario: 1) In general, which surgical treatment would you favor? Responses included mastectomy, BCS with RT, BCS without RT (DCIS scenario only), and neither procedure over the other; 2) What is the 5-year disease recurrence rate in the ipsilateral breast in these patients after a lumpectomy with clean margins and RT? Assigned ranges of $<5 \%, 5-10 \%, 11-20 \%$, and $>20 \%$ were provided as options; and 3) On average, quality of life (QOL) at 1 year after surgery is higher for which procedure? Response options were mastectomy, BCS with RT, and they confer the same QOL.

\section{Analysis}

We evaluated surgeons' responses to which treatments they favored for the two clinical scenarios. Surgeon responses were evaluated for each scenario by relative breast surgery volume and site using bivariate statistics. We tested the independent effects of selected covariates using multivariable analyses. The modeling strategies were tailored to address some limitations of power. First, for the DCIS scenario, the dependent variable was limited to BCS without RT compared with BCS with RT because very few surgeons chose the other response options (only 3 surgeons favored mastectomy and 11 favored neither procedure). Logistic regression was used to evaluate the independent effects of relative breast surgery volume, years in practice (continuous), gender, practice setting, geographic site, and patient age specified in the clinical scenario (age 65 yrs vs. $40 \mathrm{yrs}$ ) on the

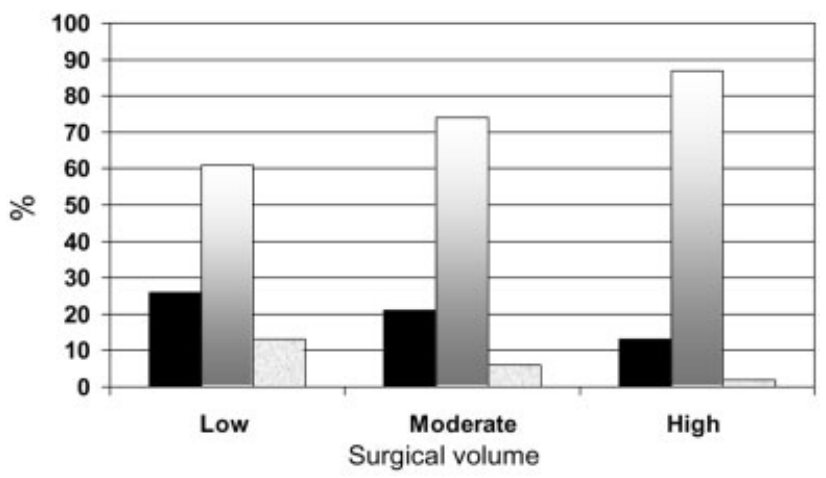

FIGURE 1. Surgical treatment favored for the invasive disease scenario, by surgical volume. Figures are unadjusted (Wald test $=14.8[P>0.001]$, controlling for yrs in practice, gender, setting, and site). Solid bar: neither; shaded bar: breast-conserving surgery; lightly shaded bar: mastectomy.

dichotomous outcome variable. Second, for the invasive scenario, we regressed three treatment response categories (favored BCS, did not favor one procedure over the other, favored mastectomy) on the selected covariates using a multinomial logistic model. Third, we evaluated surgeon perspectives on risk of disease recurrence and the QOL benefits of surgical treatment for both scenarios. The response categories for the QOL item were restricted to BCS versus "they confer the same quality of life" because very few surgeons indicated that mastectomy was superior to BCS with regard to QOL. Logistic regression was used to evaluate the independent association of selected covariates with these response categories.

\section{RESULTS}

Table 1 shows the population characteristics. The mean age of the patients was 49.4 years; $14.4 \%$ were female; and the mean number of years in practice was 17.2. On average, surgeons reported that they devoted $31.3 \%$ of their total practice to breast carcinoma and approximately one-half of their total practice time was devoted to noncancer-related procedures. There was a broad distribution of annual procedure volume by surgeon: $28.5 \%$ reported that they performed $\leq 20$ definitive breast surgeries per year, whereas $31.0 \%$ performed $>50$ procedures per year. Approximately one-half of surgeons practiced in a community hospital setting alone, whereas $18.8 \%$ practiced in a cancer center.

Surgeons were asked about which procedure they favored for the patient described in the invasive disease scenario: $73.3 \%$ favored BCS with RT; $7.0 \%$ favored mastectomy; and $19.7 \%$ did not favor either procedure. However, Figure 1 shows that there was a strong association between surgeon opinion and 


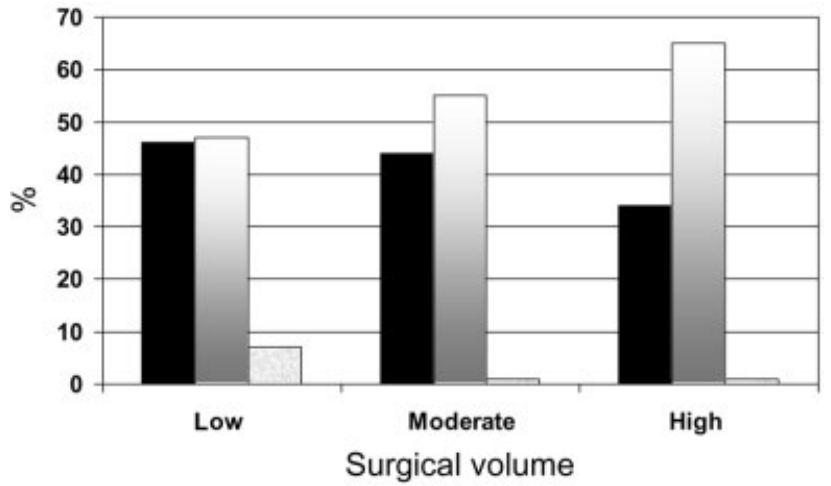

FIGURE 2. Quality of life at 1 year is higher for which treatment (invasive disease scenario)? Figures are unadjusted (Wald test $=5.1[P=0.03]$, controlling for yrs in practice, gender, setting, and site). Solid bar: same; shaded bar: breast-conserving surgery; lightly shaded bar: mastectomy.

breast surgery volume. Compared with low volume surgeons, high volume surgeons were more likely to favor BCS with RT and less likely to favor mastectomy or to not favor one procedure over the other $(60.8 \%$, $74.0 \%$, and $87.2 \%$ favoring BCS for low, moderate, and high volume surgeons, respectively; Wald test $=23.0$, $P<0.001$ controlling for yrs in practice, gender, hospital practice setting, and site). Neither hospital practice setting nor patient age described in the scenario was significantly associated with surgeon opinion. However, compared with their male colleagues, female surgeons were somewhat more likely to favor neither procedure over the other (odds ratios $[\mathrm{OR}]=$ 2.1; 95\% confidence interval [95\% CI], 0.9-4.9 controlling for relative breast surgery volume, yrs in practice, and site).

Variations in surgeon opinions regarding surgery options in the invasive clinical scenario did not appear to be related to variations in perspectives concerning recurrence of disease. Approximately one-third of surgeons estimated a 5-year disease recurrence risk of $<5 \%$ after BCS with RT and clean margins; $53.4 \%$ estimated this risk at $5-10 \%$; and $14.4 \%$ estimated the risk at $>10 \%$. Risk estimates were not associated with relative breast surgery volume or with surgeons' treatment preferences. For example, of the surgeons estimating the 5-year risk of local disease recurrence as $<5 \%, 71.7 \%$ preferred BCS compared with $68.6 \%$ of surgeons who estimated the risk of local disease recurrence as $>10 \%(P=0.591)$.

However, surgeon preferences for surgery options in the invasive clinical scenario were strongly associated with perceptions about the relative treatment benefits related to QOL. Surgeons who favored BCS were much more likely to perceive greater QOL benefits for BCS versus mastectomy (85.9\%) compared
Age 40 yrs

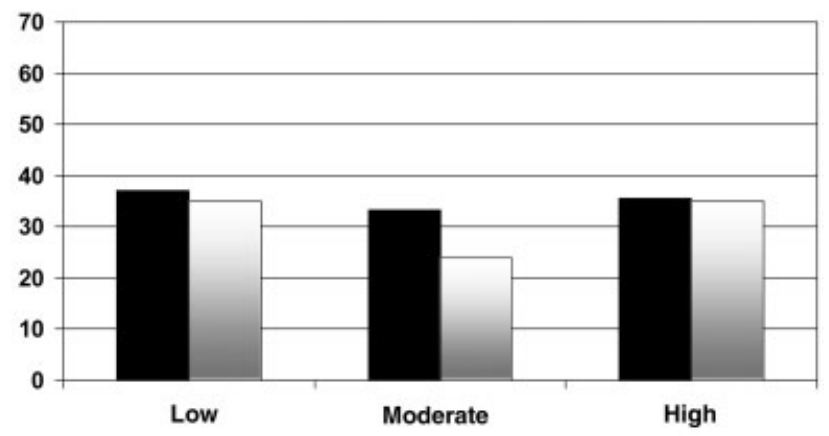

Age 65 yrs

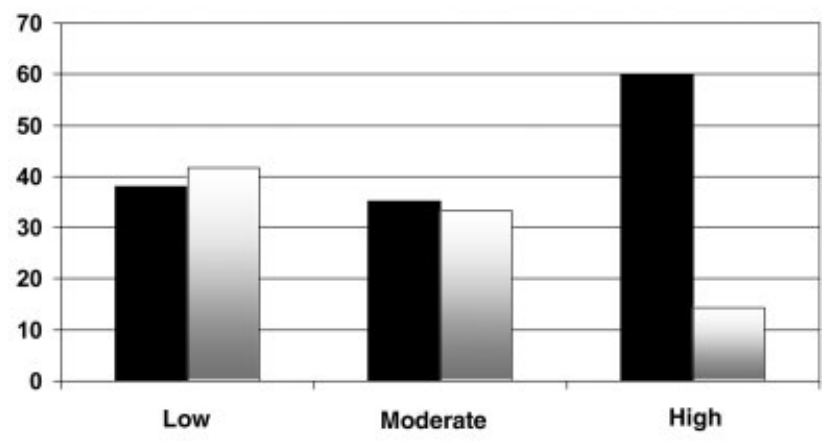

Surgical volume

FIGURE 3. Percentage of surgeons who favored breast-conserving surgery without RT for patients with ductal carcinoma in situ. Top panel: $n=186$, Wald test $=0.5(P=0.79)$, for surgeon volume $\times$ site interaction, controlling for years in practice, gender, and setting. Bottom panel; $n=168$, Wald test $=6.8$ $(P=0.034)$, for surgeon volume $\times$ site interaction, controlling for years in practice, gender, and setting. The age of patient scenario was assigned randomly. Fourteen surgeons were excluded from analysis (3 surgeons favored mastectomy and 11 surgeons did not favor 1 procedure over the other). Solid bar: Los Angeles; shaded bar: Detroit.

with surgeons who favored mastectomy (28.6\%) and those who did not favor 1 procedure over the other (60.0\%; $P<0.001)$.

Figure 2 shows that for women with invasive disease, high volume surgeons were much more likely to perceive that the QOL at 1 year is higher for women who received BCS with RT than for women who received mastectomy $(47.2 \%, 55.3 \%, 65.4 \%$ for low, moderate, and high volume surgeons, respectively; Wald test $=27.5, P<0.001$ controlling for yrs in practice, gender, hospital practice setting, and site). However, female surgeons were more likely to believe that the 2 surgical procedures conferred the same QOL at 1 year $(\mathrm{OR}=2.0 ; 95 \% \mathrm{CI}, 1.0-4.2$ controlling for 
relative breast surgery volume, yrs in practice, and site). These gender differences in perception of QOL were consistent across the DCIS and invasive carcinoma scenarios.

In the DCIS scenario, $96 \%$ of surgeons favored BCS: $35.0 \%$ favored BCS without RT; $61.0 \%$ favored BCS with RT; $3.1 \%$ (11 surgeons) did not favor 1 procedure over the other; and only $0.9 \%$ ( 3 surgeons) favored mastectomy. The difference in the proportion of surgeons favoring BCS for DCIS and those favoring BCS for invasive carcinoma was statistically significant $(P<0.001)$.

Figure 3 shows the proportion of surgeons who favored BCS without RT by relative breast surgery volume and site for the DCIS scenario. The top panel shows the results for surgeons who were randomly assigned the DCIS scenario for a patient age 40 years and the bottom panel shows the results for a patient age 65 years. Approximately one-third of the surgeons who viewed the DCIS scenario based on a 40-year-old patient favored BCS without RT and there was little difference by tumor site or volume (top panel). However, among surgeons who viewed the DCIS scenario based on a woman age 65 years (bottom panel), high volume surgeons in Los Angeles greatly favored BCS without RT $(60.6 \%)$ whereas this figure was much lower for all other surgical volume groups in both regions, especially high volume surgeons in Detroit (14.3\% favoring BCS without RT). The interaction between relative breast surgery volume and site was significant for the 65-year-old DCIS scenario (Wald test $=6.8, P=0.034$ controlling for yrs in practice, surgeon gender, and hospital practice setting).

These between-site differences did not appear to be explained by differences in surgeon perceptions regarding recurrence of disease. Indeed, for the DCIS scenario, surgeons in Los Angeles perceived higher recurrence of disease in the ipsilateral breast at 5 years after treatment with BCS with RT and clean margins compared with surgeons in Detroit. In Los Angeles, $34.5 \%$ of surgeons endorsed $a \geq 5 \%$ disease recurrence risk versus a $<5 \%$ risk compared with $23.3 \%$ of surgeons in Detroit $(P=0.012)$.

\section{DISCUSSION}

We found that that there was a wide variation in clinical volume and practice setting among a large group of surgeons who performed breast surgery in the Detroit and Los Angeles metropolitan areas in 2002. Variation in clinical experience, as measured by surgical volume, was markedly associated with surgeon opinions about treatment options. However, the type of hospital in which a surgeon practiced was not associated with a preference for BCS versus mastectomy after controlling for other factors. Previous stud- ies $^{2,24-26}$ have demonstrated that surgery in a teaching hospital or cancer center was associated with receipt of BCS. These studies did not adjust for case volume, which may account for the difference in the current study findings. For the invasive breast carcinoma scenario, surgeons in the high volume category were more likely to favor BCS with RT (vs. mastectomy or not favoring one procedure over the other) compared with their colleagues in the low and moderate volume categories. This appeared to be based partly on more favorable opinions about the QOL benefits of BCS compared with mastectomy. Our results suggest that female surgeons viewed these issues differently, as they were less inclined to favor one procedure over the other and less likely to view BCS as superior to mastectomy with regard to QOL. This view is consistent with multiple studies that have compared QOL after mastectomy and BCS. ${ }^{27-30}$

A similar observation regarding the effect of volume on the use of BCS was made by Mandelblatt et al., ${ }^{20}$ who used clinical vignettes to evaluate surgical perspectives on the treatment of invasive breast carcinoma in older women. Our population-based study differs from their hospital-based sample of attending surgeons treating Medicare beneficiaries with breast carcinoma in 1998. However, we found that the positive relation between breast carcinoma volume and the use of BCS applies to younger women as well as those in the Medicare age group. In contrast to our study, they found that female surgeons were more likely to favor BCS versus male colleagues, whereas we found that female surgeons were more neutral about BCS, favoring neither procedure over the other. However, the proportion of female surgeons in both studies was quite low $(6.6 \%$ in the Mandelblatt et al. study and $14.4 \%$ in the current study). Our observations are consistent with the report of Weinberg et al. ${ }^{31}$ who studied 2271 patients with breast carcinoma treated in a Cincinnati health plan and found that after adjustment for disease stage, those treated by male surgeons were $30 \%$ more likely to undergo BCS.

Surgical perceptions regarding the treatment of early invasive carcinoma and DCIS also differed significantly: $73 \%$ endorsed BCS for early-stage invasive carcinoma scenario versus $96 \%$ for the DCIS scenario. Although surgeons' estimates of the disease recurrence risk did not correlate with surgical treatment choice, only $1.7 \%$ of surgeons believed that the 5 -year risk of local disease recurrence for DCIS treated with BCS and RT was $>10 \%$, whereas $14.4 \%$ endorsed local disease recurrence rates $>10 \%$ for invasive carcinoma treated with lumpectomy and RT. The QOL benefit for BCS treatment of both invasive disease and DCIS was endorsed by the same proportion of surgeons. These 
findings suggest that the assumption of clinical superiority of BCS in invasive carcinoma may not be fully accepted by a significant minority (approximately one-fourth) of surgeons. The preference for BCS in DCIS compared with invasive carcinoma is somewhat puzzling. Six prospective randomized trials have demonstrated that mastectomy does not provide a survival advantage over BCS in invasive carcinoma. ${ }^{32-38}$ The findings of the current study suggest that some surgeons still endorse the concept that a larger operation (mastectomy) is more likely to result in the cure of invasive carcinoma in spite of a substantial body of data that indicates that this is not the case.

We found that there were significant differences between geographic sites with regard to perspectives about the role of RT after BCS in the DCIS scenario. Surgeons in Los Angeles were more likely than their counterparts in Detroit to endorse the use of BCS alone in the DCIS patient scenario. However, this site difference was modified by relative breast surgery volume and patient age in the clinical scenario: site differences were observed only among high volume surgeons in the scenario of an older patient with DCIS. Three prospective randomized trials have demonstrated that RT significantly reduces the risk of local disease recurrence in women with DCIS treated with BCS. However, the absolute benefit of RT is quite small in some subgroups, and there is increasing evidence that younger women have a higher risk of local failure than their older counterparts. ${ }^{39,40}$ The greater preference of high volume surgeons in Los Angeles for treatment of older women with BCS alone may reflect the influence of local opinion leaders who believe that the marginal benefit of RT is outweighed by its morbidity in patients at low risk of local disease recurrence such as elderly women with low-grade DCIS which has been widely excised. ${ }^{41-43}$ The preference of high volume surgeons in Detroit for RT may be a reflection of a strict interpretation of the results of the randomized trials that demonstrate a reduction in local disease recurrence in all patient subgroups treated with RT. The reasons for the differences in preference for the use of RT warrant further investigation.

\section{Limitations}

Our estimates may have been biased by nonresponse and we had little information regarding nonrespondent surgeons. However, the response rate of $80 \%$ was excellent for a physician survey. We believe that the surgeon sample is representative of all surgeons practicing breast carcinoma care in the two metropolitan areas during the study period because they were identified through a population-based sample of patients. However, we likely missed some surgeons, particularly those with a low breast surgery volume. Also, the scenarios we developed were based on extensive feedback and piloting among surgeons. However, they were hypothetical and thus may not exactly reflect surgeons' opinions expressed during direct patient interactions. We purposely limited the information in the scenarios to reflect what is often available at the time of the first consultation for surgical treatment. Both scenarios describe patients with no obvious contraindications to $\mathrm{BCS},{ }^{44}$ and there is no reason to expect that providing additional information would change our findings related to surgeon or practice characteristics. Variations in the information available at the time of consultation may also limit the generalizability of the scenarios to the decision-making process. Practice information was derived from self-report and, thus, subject to respondent recall. However, surgeon recall of procedure volume was highly correlated with the number of patients treated in our patient study.

\section{Conclusions}

The results of the current study have important implications for patient care and policy. Variation in surgeon opinion with regard to local therapy reflects legitimate clinical uncertainty and/or disagreement about the marginal benefits of alternative treatment options. These results suggest that these variations are partly related to surgeon characteristics. We found that relative breast surgery volume and surgeon gender were important independent correlates of surgeon attitudes toward approaches to treatment. Higher volume surgeons appeared to more proactively endorse current clinical guidelines that favor BCS over mastectomy, which may be explained in part by the greater belief that BCS confers higher patient QOL than mastectomy. Our results suggest that female surgeons view the treatment issues differently than their male counterparts because they appear less convinced of the superior QOL after BCS. This may reflect gender differences in patient communication and feedback or gender differences in the relative weight given to the importance of preserving the breast versus the negative impact of local disease recurrence and the need for additional surgery on QOL.

Regardless of the etiology of variation in surgeon attitudes toward treatment, patients should be aware that surgeon perspectives and opinions may be influenced by surgical experience and gender, especially regarding how strongly surgeons may favor BCS and how strongly surgeons may favor RT after BCS for patients with DCIS. Patients who are advised to undergo a particular procedure without a full discussion of treatment options should request a second opinion if a clear medical rationale for the recommendation is not provided. 


\section{REFERENCES}

1. Farrow DC, Hunt WC, Samet JM. Geographic variation in the treatment of localized breast cancer. $N$ Engl J Med. 1992;326:1097-1101.

2. Nattinger AB, Gottlieb MS, Veum J, Yahnke D, Goodwin JS. Geographic variation in the use of breast-conserving treatment for breast cancer. N Engl J Med. 1992;326:1102-1107.

3. Ernster VL, Barclay J, Kerlikowske K, Grady D, Henderson C. Incidence of and treatment for ductal carcinoma in situ of the breast. JAMA. 1996;275:913-918.

4. Mandelblatt JS, Hadley J, Kerner JF, et al. Patterns of breast carcinoma treatment in older women: patient preferences and clinical and physical influences. Cancer. 2000;89:561-573.

5. Thompson TA, Pusic A, Kerrigan CL, et al. Surgeon perspectives on surgical options for early-stage breast cancer. Plast Reconstr Surg. 2000;105:910-918.

6. Wennberg JE. Unwarranted variations in health care delivery: implications for academic medical centres. BMJ. 2002; 325:961-964.

7. Kotwall C, Brinker C, Covington DL, et al. Local and national trends over a decade in the surgical treatment of ductal carcinoma in situ. Am J Surg. 2003;186:723-728.

8. Baxter NN, Virnig BA, Durham SB, Tuttle TM. Trends in the treatment of ductal carcinoma in situ of the breast. J Natl Cancer Inst. 2004;96:443-448.

9. White J, Morrow M, Moughan J, et al. Compliance with breast-conservation standards for patients with early-stage breast carcinoma. Cancer. 2003;97:893-904.

10. Stafford D, Szczys R, Becker R, Anderson J, Bushfield S. How breast cancer treatment decisions are made by women in North Dakota. Am J Surg. 1998;176:515-519.

11. Institute of Medicine Report. Ensuring quality cancer care. In: Hewitt M, Simone JV, editors. National Cancer Policy Board, Institute of Medicine Report and National Research Council. Washington, DC: National Academy Press, 1999:97.

12. Kotwall CA, Maxwell JG, Covington DL, Churchill P, Smith SE, Covan EK. Clinicopathologic factors and patient perceptions associated with surgical breast-conserving treatment. Ann Surg Oncol. 1996;3:169-175.

13. Smitt MC, Heltzel M. Women's use of resources in decisionmaking for early-stage breast cancer: results of a community-based survey. Ann Surg Oncol. 1997;4:564-569.

14. Gemer L. Breast conservation therapy for breast cancer treatment in Brown County. West Med J. 1999;98:50-55.

15. Nold RJ, Beamer RL, Helmer SD, McBoyle MF. Factors influencing a woman's choice to undergo breast-conserving surgery versus modified radical mastectomy. Am J Surg. 2000;180:413-418.

16. Molenaar S, Oort F, Sprangers M, et al. Predictors of patients' choices for breast-conserving therapy or mastectomy: a prospective study. Br J Cancer. 2004;90:2123-2130.

17. Morris J, McNoe B, Elwood JM, Packer S. Breast cancer surgery in New Zealand: consensus or variation? $N Z$ Med J. 1997;110:53-56.

18. Spyrou G, Titley O, Cerqueiro J, Fatah M. A survey of general surgeons' attitudes towards breast reconstruction after mastectomy. Ann R Coll Surg Engl. 1998;80:178-183.

19. Porter G, McMulkin-Tait H. Practice patterns in breast cancer surgery: Canadian perspective. World J Surg. 2004;28:8086.

20. Mandelblatt JS, Berg CD, Meropol NJ, et al. Measuring and predicting surgeons' practice styles for breast cancer treatment in older women. Med Care. 2001;39:228-242.
21. Katz SJ, Lantz PM, Janz NK, et al. The role of patient involvement in surgical treatment decisions for breast cancer. J Clin Oncol. 2005;23:5526-5533.

22. Dillman DA. Mail and telephone surveys. New York: John Wiley and Sons, Inc., 1978.

23. Anema MG, Brown BE. Increasing survey responses using the total design method. J Contin Educ Nurs. 1995;26:109-114.

24. Samet J, Hunt W, Farrow D. Determinants of receiving breastconserving surgery. The Surveillance, Epidemiology, and End Results Program, 1983-1986. Cancer. 1994;73:2344-2351.

25. Guadagnoli E, Weeks JC, Shapiro CL, Gurwitz JH, Borbas C, Soumerai SB. Use of breast-conserving surgery for treatment of stage I and stage II breast cancer. J Clin Oncol. 1998;16:101-106.

26. Morrow M, White J, Moughan J, et al. Factors predicting the use of breast-conserving therapy in stage I and II breast carcinoma. J Clin Oncol. 2001;19:2254-2262.

27. Fallowfield LJ, Hall A, Maguire P, et al. Psychological outcomes of different treatment policies in women with early breast cancer outside a clinical trial. BMJ. 1990;301:575-580.

28. Pozo C, Carver CS, Noriega V, et al. Effects of mastectomy versus lumpectomy of emotional adjustment to breast cancer: a prospective study of the first year postsurgery. J Clin Oncol. 1992;10:1292-1298.

29. Nissen MJ, Swenson KK, Ritz LJ, Farrell JB, Sladek ML, Lally RM. Quality of life after breast carcinoma surgery. Cancer. 2001;91:1238-1246.

30. Janz NK, Mujahid M, Lantz PM, et al. Population-based study of the relationship of treatment and sociodemographics on quality of life after breast cancer. Qual Life Res. 2005;14:14671479.

31. Weinberg E, Woods S, Grannan K, Hendy MP. The influence of gender of the surgeon on surgical procedure preference for breast cancer. Am Surg. 2002;68:398-400.

32. Arriagada R, Le MG, Rochard F, Contesso G. Conservative treatment versus mastectomy in early breast cancer: patterns of failure with 15 years of follow-up data. Institute GustaveRoussy Breast Cancer Group. J Clin Oncol. 1996;14:1558-1564.

33. Veronesi U, Cascinelli N, Mariani L, et al. Twenty-year follow-up of a randomized study comparing breast-conserving surgery with radical mastectomy for early breast cancer. N Engl J Med. 2002;347:1227-1232.

34. Fisher B, Anderson S, Bryant J, et al. Twenty-year follow-up of a randomized trial comparing total mastectomy, lumpectomy and lumpectomy plus irradiation for the treatment of invasive breast cancer. $N$ Engl J Med. 2002;347:1233-1241.

35. Poggi MM, Danforth DN, Sciuto LC, et al. Eighteen-year results in the treatment of early breast carcinoma with mastectomy versus breast conservation therapy. The National Cancer Institute Randomized Trial. Cancer. 2003;98:697-702.

36. Van Dongen JA, Voogd AC, Fentiman IS, et al. Long-term results of a randomized trial comparing breast-conserving therapy with mastectomy: European Organization for Research and Treatment of Cancer 10801 trial. J Natl Cancer Inst. 2000;92:1143-1150.

37. Blichert-Toft M, Rose C, Andersen JA, et al. Danish randomized trial comparing breast conservation therapy with mastectomy: six years of life-table analysis. Danish breast cancer cooperative group. J Natl Cancer Inst Monogr. 1992;11:19-25.

38. Frykberg ER, Bland KI. In situ breast carcinoma. Adv Surg. $1993 ; 26: 29-72$ 
39. Bijker N, Peterse JL, Duchateau L, et al. Risk factors for recurrence and metastasis after breast-conserving therapy for ductal carcinoma-in-situ: analysis of European Organization for Research and Treatment of Cancer trial 10853. J Clin Oncol. 2001;19:2263-2271.

40. Fisher B, Land S, Mamounas E, Dignam J, Fisher ER, Wolmark N. Prevention of invasive breast cancer in women with ductal carcinoma in situ: an update of the National Surgical Adjuvant Breast and Bowel Project Experience. Semin Oncol. 2001;28:400-418.

41. Lagios MD, Silverstein MJ. Ductal carcinoma in situ. The success of breast conservation therapy: a shared experience of two single institutional nonrandomized prospective studies. Surg Oncol Clin North Am. 1997;6:385-392.

42. Silverstein MJ. Ductal carcinoma in situ of the breast: controversial issues. Oncologist. 1998A;3:94-103.

43. Silverstein MJ. Fortnightly review: ductal carcinoma in situ of the breast. BMJ. 1998B;317:734-739.

44. Morrow M, Bucci C, Rademaker A. Medical contraindications are not a major factor in the underutilization of breast conserving surgery. J Am Coll Surg. 1998; 186:269-274. 